\title{
ChemComm
}

Cite this: Chem. Commun., 2013, 49, 7985

Received 26th May 2013, Accepted 3rd July 2013

DOI: $10.1039 /$ c3cc43950j

www.rsc.org/chemcomm

\section{Recent advances in stereoselective bromofunctionalization of alkenes using $\mathrm{N}$-bromoamide reagents}

\author{
Chong Kiat Tan and Ying-Yeung Yeung*
}

\begin{abstract}
Bromination reactions have been made a lot more convenient since the invention of $\mathrm{N}$-bromoamide reagents. These reagents are more easily handled when compared to molecular bromine. In comparison to other halogens, brominating reagents sit in between chlorine and iodine on the reactivity scale, giving them an advantage in some cases. Recently, several important advances in enantioselective bromofunctionalization of alkenes using such reagents have been reported. This article will highlight the challenges and methods to surmount these problems. In addition, this article will also show the use of $\mathrm{N}$-bromoamide reagents in expanding the scope of diastereoselective bromofunctionalization of alkenes. Examples include bromine initiated cyclic ether cascades and novel multicomponent reactions (MCRs).
\end{abstract}

\section{Introduction}

Bromination reactions have been a workhorse in organic synthesis. However the use of bromine as a reagent itself is not entirely convenient due to its high reactivity, volatility and toxicity. The invention of $\mathrm{N}$-bromoamide reagents like $\mathrm{N}$-bromosuccinimide $(\mathrm{NBS})^{1}$ (Fig. 1) for example has greatly enhanced the utility of bromination reactions due to its safe and easy handling. In addition to NBS, a number of commercially available $\mathrm{N}$-bromoamide reagents exist including $N$-bromophthalimide (NBP),

Department of Chemistry, NUS, 3 Science Drive 3, 11754, Singapore.

E-mail: chmyyy@nus.edu.sg; Fax: +65-6779-1691; Tel: +65-6516-7760
$\mathrm{N}$-bromoacetamide (NBA) and the more reactive 1,3-dibromo5,5-dimethydantoin (DBDMH). In terms of halogen reactivity, bromine is more reactive than chlorine but less reactive than iodine towards electrophilic halogenation of alkenes. ${ }^{2}$ In terms of the leaving group ability of the halogens in the products, alkyl bromides offer higher reactivity towards $\mathrm{S}_{\mathrm{N}} 2$ displacement than alkyl chlorides. In addition, alkyl bromides are also more stable towards unwanted nucleophilic displacement when compared to alkyl iodides. ${ }^{3}$

Reactions ranging from the radical bromination of allylic ${ }^{4}$ and benzylic ${ }^{5}$ positions to electrophilic bromination of carbonyl compounds, $^{6}$ aromatic ${ }^{7}$ and heterocyclic ${ }^{8}$ compounds are just some examples of the bromination reactions that are

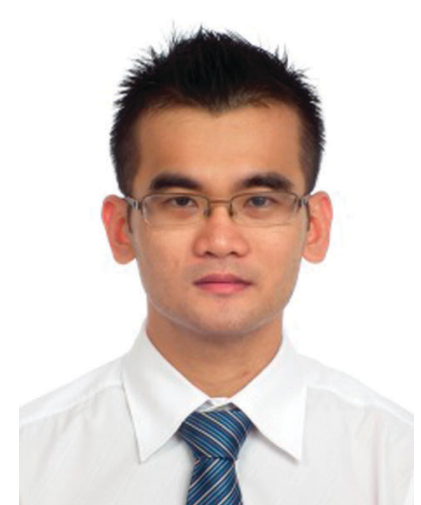

Chong Kiat Tan
Chong Kiat Tan is a final year PhD candidate at the Department of Chemistry at the National University of Singapore. He obtained his BSc (Hons) from the same university. His research interests include organic synthesis and the development of asymmetric organocatalysis.

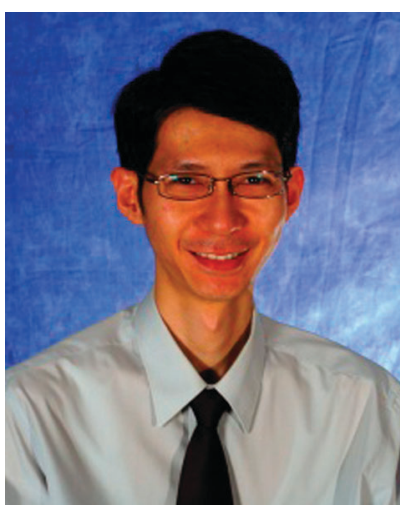

Ying-Yeung Yeung
Ying Yeung Yeung received his BSc (2001) at The Chinese University of Hong Kong. He continued his graduate research in the same university under the supervision of Prof. Tony K. M. Shing. After four years research dedicated to natural product synthesis, Dr Yeung moved to USA to conduct postdoctoral research with Prof. E. J. Corey at Harvard University. In 2008, he joined National University of Singapore, Department of Chemistry, as an assistant professor. His research interests include asymmetric catalysis, green oxidation, and methodology development. 


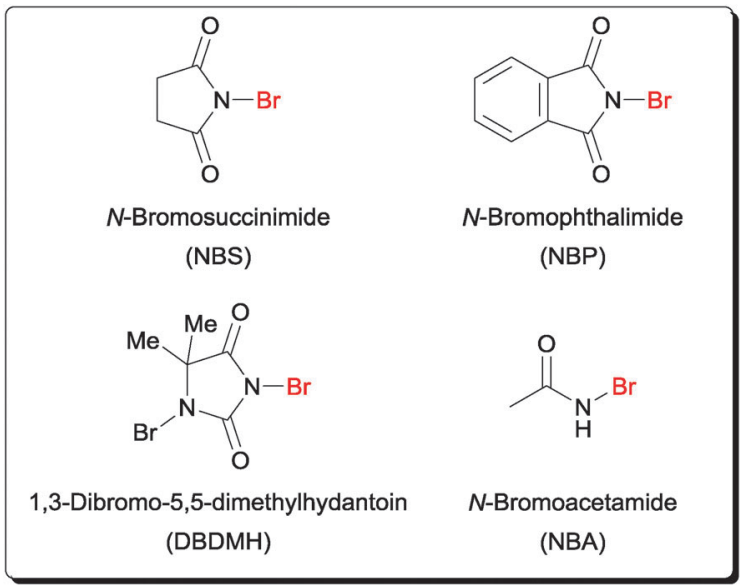

Fig. 1 Examples of $\mathrm{N}$-bromoamide reagents.

possible with such reagents. However, this feature article will only review selected examples from the recent advances of the electrophilic bromofunctionalization of alkenes starting from 2005.

The generally accepted mechanism of such reactions begins with the formation of the bromonium ion once the electrophilic bromine source comes into contact with the alkene. Such bromonium ions were observed in liquid $\mathrm{SO}_{2}$ in a series of NMR studies performed by Olah et al. ${ }^{9}$

Subsequently, this reactive bromonium ion can be further captured by a compatible nucleophile (Scheme 1). Since products that contain an anti-relationship between the bromide and nucleophile are generally obtained, the nucleophile is believed to open the bromonium ring in an $\mathrm{S}_{\mathrm{N}} 2$ fashion. ${ }^{10}$

Bromofunctionalization of alkenes generally affords products of high diastereoselectivity, as suggested by the proposed mechanism in Scheme 1.

In recent years a renewed focus has been cast on the long standing challenge of enantioselective bromofunctionalization of alkenes. More broadly, the solving of this long standing problem should be addressed under the umbrella of enantioselective halofunctionalization of alkenes. Several reviews on this topic are already in the literature. ${ }^{11}$

This article will only limit its coverage to the electrophilic bromofunctionalization of alkenes using $N$-bromoamide reagents. The article consists of two parts: part one will review the recent progress in the catalytic enantioselective bromofunctionalization of alkenes using $N$-bromoamide reagents, and the second part will address the other significant progress in diastereoselective bromofunctionalization of alkenes. Efforts from our own laboratory in these two fields will be described as well.

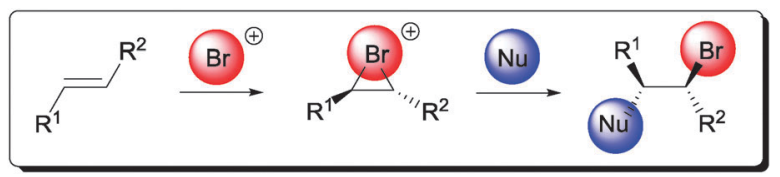

Scheme 1 General mechanism of the electrophilic bromofunctionalization of alkene.

\section{Part I - recent progress in enantioselective bromofunctionalization of alkenes}

The enantioselective bromofunctionalization or more broadly halofunctionalization of alkenes has been an area of intensive research in recent years. Many of the difficulties in achieving bromofunctionalization with a useful level of enantioselectivity have been worked out mechanistically by Brown et al. as well as Denmark et al.

Brown and co-workers were the first to demonstrate that a rapid and degenerative olefin-to-olefin transfer exists between the bromonium ion and free alkenes in NMR studies (Scheme 2). ${ }^{12}$ The rate of this olefin-to-olefin transfer process was also found to be competitive with the nucleophilic capture of the bromonium ion. With the existence of such a rapid bromonium transfer mechanism, one can rationalize the possibility of racemization of an enantioenriched bromonium ion. This possible racemization mechanism thus makes the development of the enantioselective bromofunctionalization of alkene a challenging task.

The next important mechanistic rationalization of the problem of enantioselective bromofunctionalization was demonstrated in a series of acetolysis experiments conducted by Denmark and co-workers. ${ }^{13}$ The experiments simulate the environment where the configurational stability of both bromonium and chloronium ions can be determined in the presence of both a nucleophile and excess olefin. The $\beta$-halo sulfonates $\mathbf{1 a}$ and $\mathbf{1 b}$ can generate the respective bromonium and chloronium ions in strongly ionizing media. The rates of racemization by excess (E)-4-octene (via olefin-to-olefin transfer, Scheme 2) can thus be compared to the rate of intermolecular nucleophilic capture of the halonium ion.

They discovered that the addition of 4-octene into a solution of bromotosylate 1a resulted in the erosion of enantiospecificity in the highly polar hexafluoroisopropyl alcohol (HFIP) solvent medium (Scheme 3). The main factors affecting the erosion of enantiospecificity were identified to be the concentration of the olefin (4-octene) and the counter cation of the acetate. The observation for bromotosylate stands in complete contrast to the analogous case of chlorotriflate $\mathbf{1} \mathbf{b}$, where enantiospecificity was preserved even in the presence of 4-octene. These results established the relative stability of the chloronium ion compared to the bromonium ion.

The above two observations suggest the inherent difficulty of forming a stable and chiral bromonium ion due to the presence of a rapid and degenerate olefin-to-olefin transfer mechanism. Nonetheless, some solutions to this problem have been unravelled in recent years and will be described in the following sections. It is noteworthy that breakthroughs in chloro- $^{14}$ and

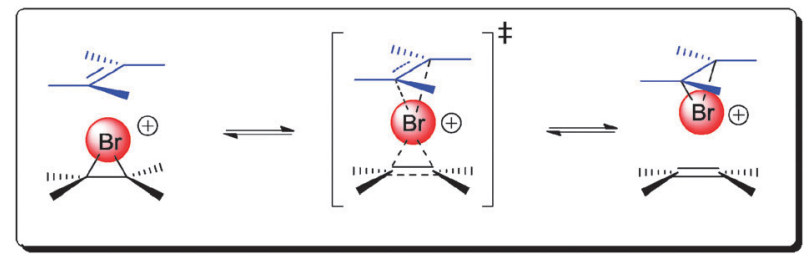

Scheme 2 Degenerate olefin-to-olefin transfer of bromonium. 


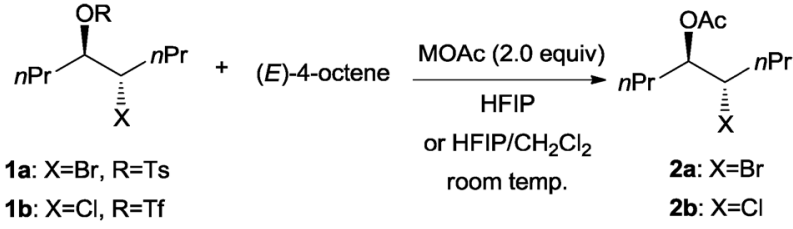

\begin{tabular}{|c|c|c|c|}
\hline$x$ & $M$ & $\begin{array}{l}\text { Equiv of } \\
(E) \text {-4-octene }\end{array}$ & es (\%) \\
\hline $\mathrm{Br}$ & $\mathrm{Na}$ & 0.0 & 100 \\
\hline $\mathrm{Br}$ & $\mathrm{Na}$ & 1.0 & 28 \\
\hline $\mathrm{Br}$ & $n \mathrm{Bu}_{4} \mathrm{~N}$ & 1.0 & 81 \\
\hline $\mathrm{Cl}$ & $n \mathrm{Bu}_{4} \mathrm{~N}$ & 1.0 & 100 \\
\hline
\end{tabular}

Reactions were conducted at $1.0 \mathrm{M}$ substrate concentration.

Scheme 3 Effect of halogen, counter cation, and concentration of alkene on the erosion of enantiospecificity.

iodofunctionalization ${ }^{15}$ of alkenes were also achieved concurrently in recent years.

\subsection{Enantioselective intramolecular bromofunctionalization of alkenes}

One successful report of the enantioselective bromofunctionalization of alkenes was disclosed by the laboratory of Tang and co-workers. ${ }^{16}$ Their solution to this problem was the use of the quinine derived tosyl urea 3 as the chiral organocatalyst for the bromolactonization of $Z$-1,3-enynes (Scheme 4).

The design of catalyst 3 was preceded by a previous work from their laboratory which demonstrated the competence of 1,4-diazabicyclo-[2.2.2] octane (DABCO) as a bromolactonization catalyst. ${ }^{17}$ In this report they proposed a dual activation mode where NBS is activated via a hydrogen bonding with the NH groups of the urea and the carboxylate anion is bound to the

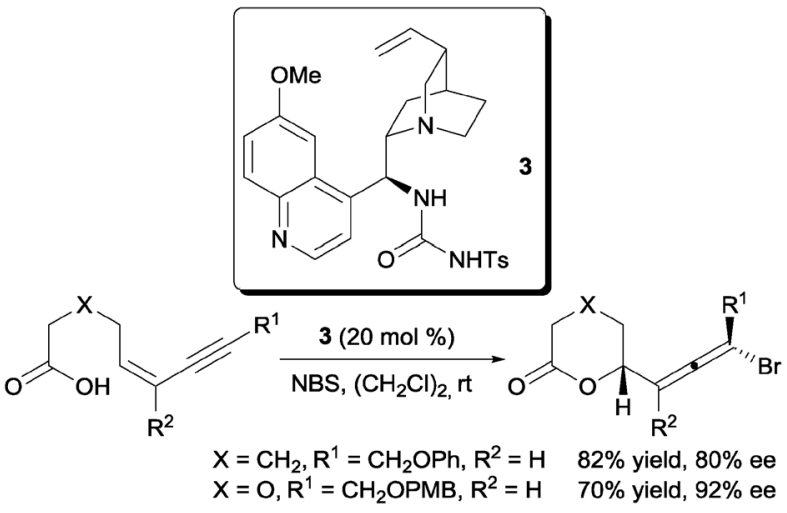

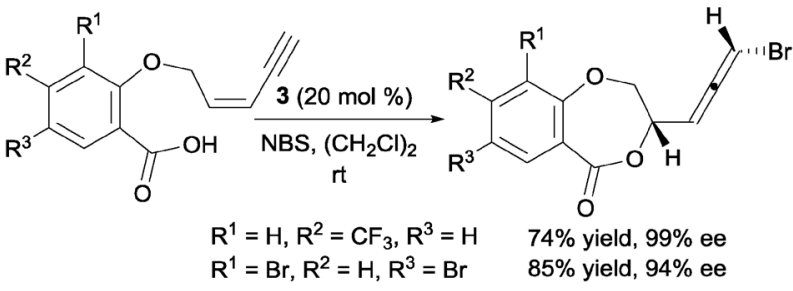

Scheme 4 Enantioselective bromolactonization of Z-1,3-enynes. quinuclidine of the quinine scaffold. This proposed mechanism was also supported by the change in enantioselectivities of the bromolactonization with various halogen sources especially between NBS and tetrabromocyclohexadienone. ${ }^{16 b}$

Another competent chiral organocatalyst came in the form of the $C_{3}$-symmetric trisimidazoline 4 reported by Fujioka $e t$ al. (Scheme 5). ${ }^{18}$ Using their protocol, a variety of 5-aryl-substituted hexenoic acids are amendable to enantioselective bromolactonization. Their substrate scope includes 1,1-disubstituted, trisubstituted, and tetrasubstituted hexenoic acids. Mechanistically, they found the presence of the three imidazoline units to be crucial to achieving high enantioselectivity. The use of both the mono- and di-imidazoline variants of catalyst $\mathbf{4}$ resulted in much lower enantioselectivities. Additionally, NMR studies conducted by the group suggest the probable templating of three units of hexenoic acids to one unit of catalyst $\mathbf{4}$ as shown in model $\mathbf{A}$ (Scheme 5).

Another different solution was offered by our laboratory with a class of amino-thiocarbamate catalysts. The first of this class of amino-thiocarbamate came with a cinchona alkaloid scaffold (Scheme 6). After extensive studies, two catalyst tuning sites
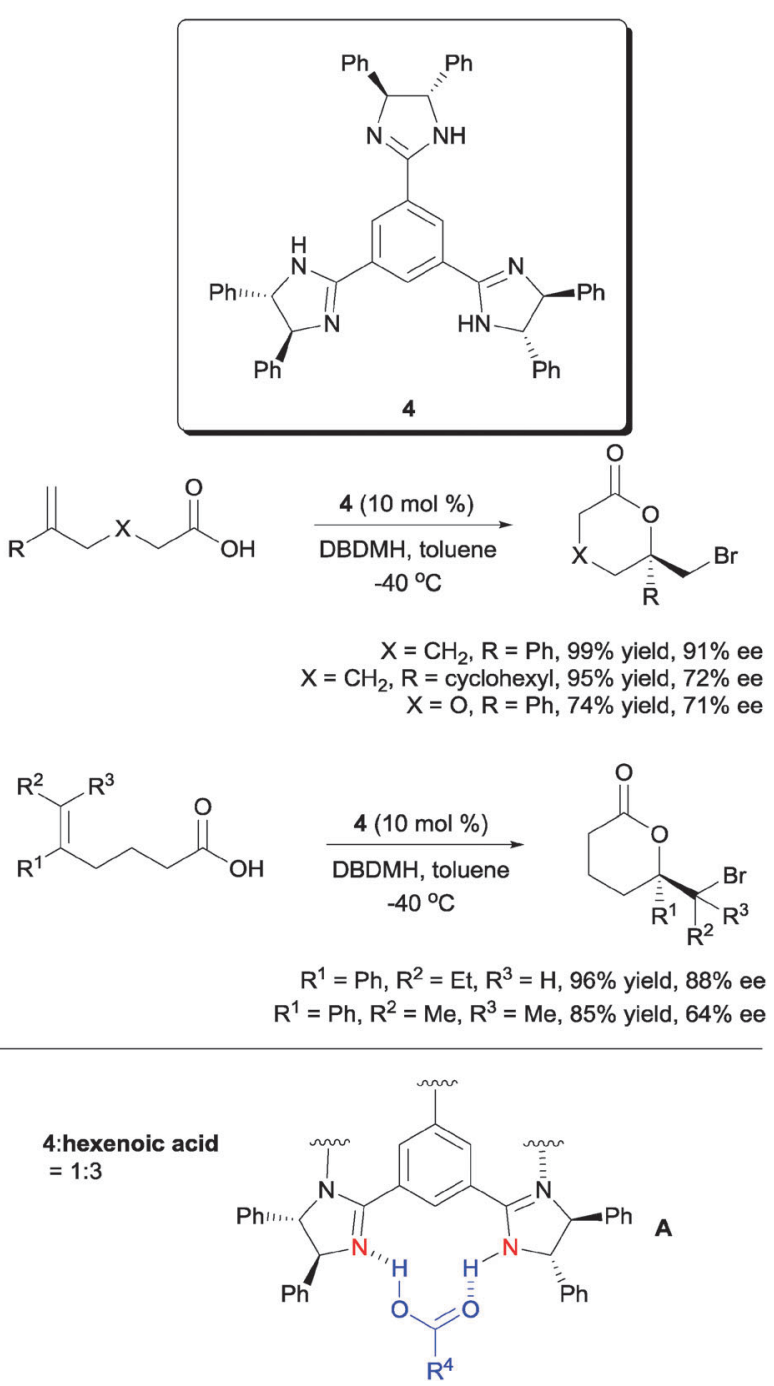

Scheme 5 Enantioselective bromolactonization with $C_{3}$-symmetric trisimidazoline. 

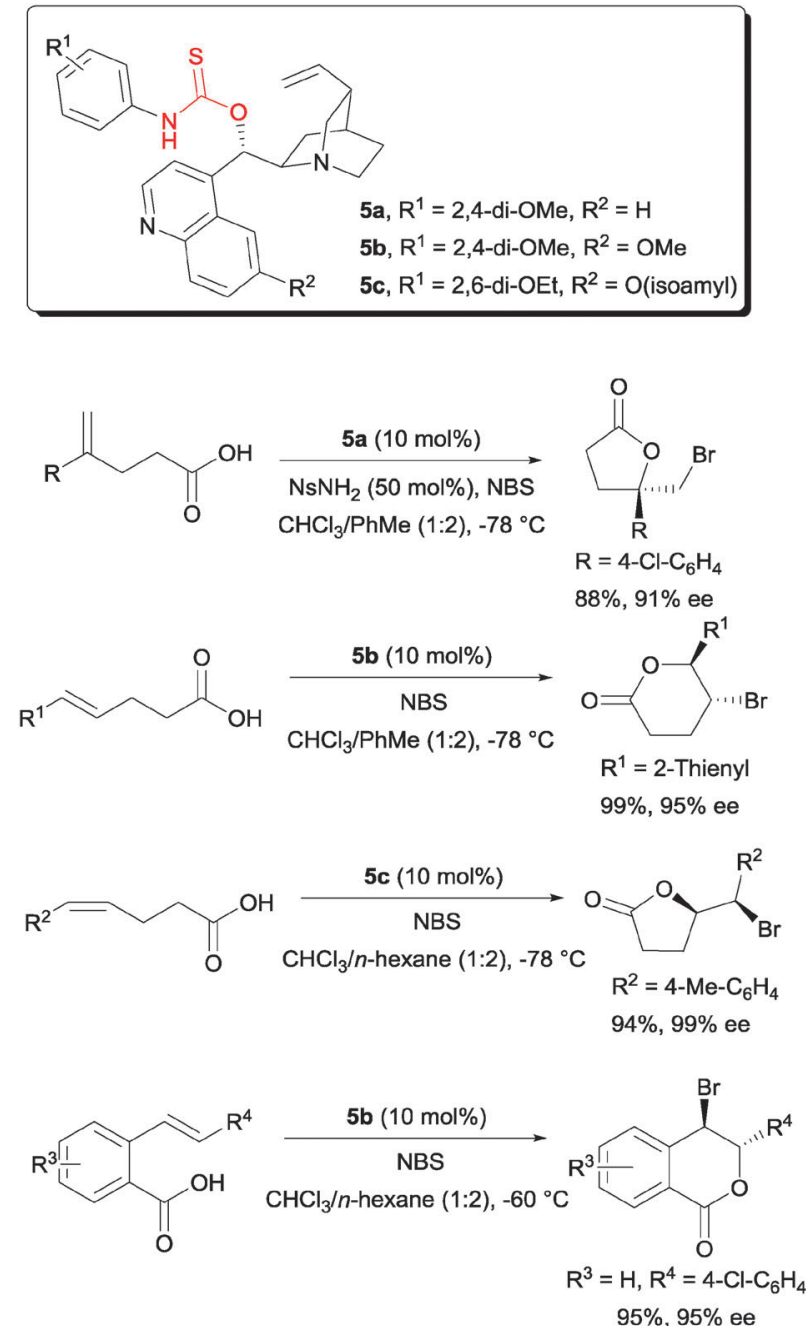

Scheme 6 Enantioselective bromolactonization with amino-thiocarbamate catalysts.

were discovered. These are the $N$-aryl substituent indicated as $\mathrm{R}^{1}$ and the $O$-alkoxy substituent at the quinoline unit indicated as $\mathrm{R}^{2}$. The tuning of these sites allowed a number of alkenoic acids to be accommodated into this protocol. These include 1,1-disubstituted pentenoic acid, ${ }^{19}$ both $E$ and $Z$-pentenoic acid, ${ }^{20,21}$ and the styrene type carboxylic acid. ${ }^{22}$

While the above example demonstrated the essentiality of using $O$-alkyl thiocarbamate on a cinchona alkaloid scaffold, subsequent work shown that $S$-alkyl thiocarbamates are also competent chiral organocatalysts on a proline backbone (Scheme 7). ${ }^{23}$ In one of our studies, $S$-alkyl thiocarbamate catalyst 6 was able to serve as a chiral catalyst for the bromolactonization of hexenoic acids. On the other hand, $O$-alkyl thiocarbamate 7 could catalyze the enantioselective bromolactonization of pentenoic acids. An additional motivation for carrying out the work on a proline scaffold is that both enantiomers of proline are available. In contrast, only pseudo-enantiomeric pairs of cinchona alkaloids are commercially available. The magnitudes of enantioselectivity of some bromolactones are not equal when a pseudo-enantiomeric pair of cinchona alkaloid derived thiocarbamates were used in some specific studies. ${ }^{21,22}$

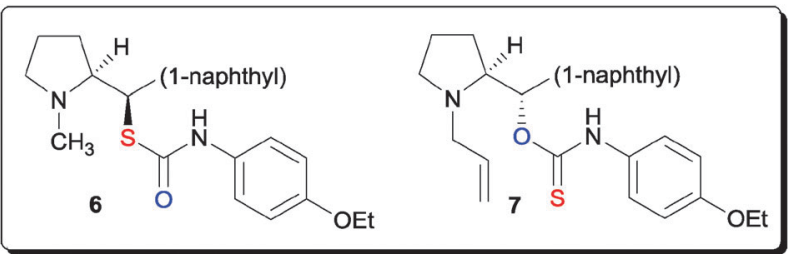

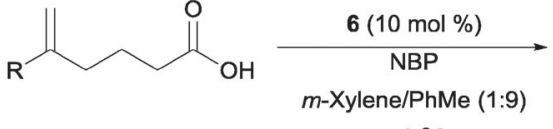

$-78^{\circ} \mathrm{C}$

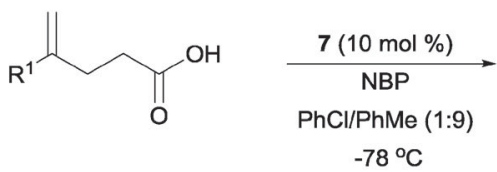

$-78^{\circ} \mathrm{C}$

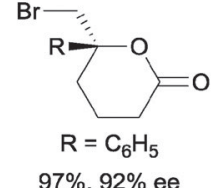

$92 \%$ ee

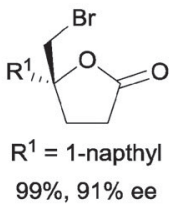

Scheme 7 Enantioselective bromolactonization with both $O$ - and S-alkyl aminothiocarbamates.
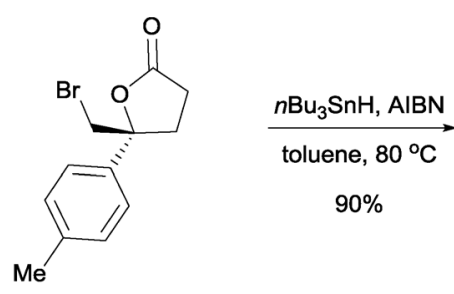

$90 \%$

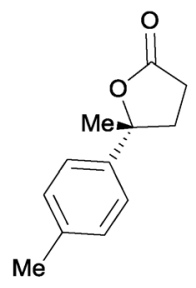

$(R)-(+)$-Boivinianin A
Scheme 8 Synthesis of $(R)-(+)$-boivinianin A from chiral bromolactone.

The enantioenriched bromolactones can be converted into valuable intermediates. In one example, we were able to perform the debromination of a bromolactone into $(R)-(+)$-boivinianin $\mathrm{A}$ (Scheme 8). ${ }^{24}$ The enantiomer of $(R)-(+)$-boivinianin A can readily be accessed by using the enantiomer of the proline based catalyst 7 .

The amino-thiocarbamate catalysts were also found to be able to perform the enantioselective bromoaminocyclization of a variety of olefinic amides (Scheme 9). In the series of studies, amides 9, 11 and 13 were found to be possible substrates for achieving high enantioselectivities. $^{25}$ One of the essential requirements for this reaction is the presence of the nosyl substituents. The $\mathrm{NH}$ of the 4-nosyl amide is then sufficiently acidic for the reaction to take place smoothly in an enantioselective fashion. The resulting enantioenriched bromo-pyrrolidine and piperidine products are useful building blocks of various pharmaceuticals.

Mechanistically, the above reactions catalyzed by aminothiocarbamates were proposed to proceed via a dual activation mode. The sulfur atom in the catalyst is believed to act as a Lewis base activator of bromine, ${ }^{26}$ while the amine may interact with the nucleophile via an acid-base interaction (Model B, Scheme 10). ${ }^{19}$

Sulfur or more broadly chalcogens have been known to form stable 1:1 molecular adducts with bromine. ${ }^{27}$ Denmark et al. have also demonstrated the competence of sulfur-containing compounds as both bromolactonization and bromoetherification catalysts. ${ }^{28}$ 


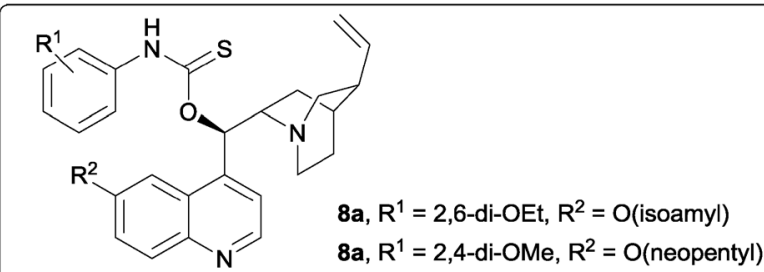

$\mathrm{R}^{1}$<smiles>[R][R]O[Ga]=[R4]=[R4]</smiles>

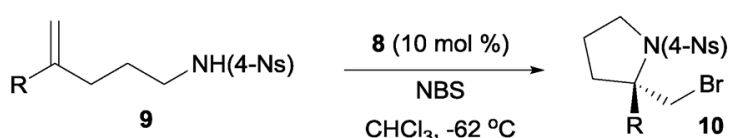
$\mathrm{R}=4-\mathrm{Cl}-\mathrm{C}_{6} \mathrm{H}_{4}$ $98 \%, 98 \%$ ee<smiles>[R]C=CCCCNC#N</smiles><smiles>[R]C1NCCC[C@H]1Br</smiles>
$\mathrm{R}^{1}=3-\mathrm{Me}-\mathrm{C}_{6} \mathrm{H}_{4}$ $98 \%, 90 \%$ ee
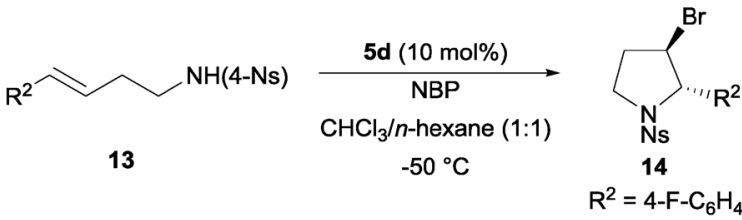

$89 \%, 91 \%$ ee

Scheme 9 Enantioselective bromoaminocyclization with amino-thiocarbamates

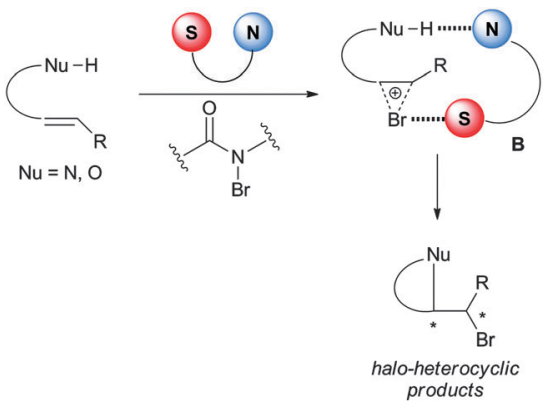

Scheme 10 Proposed mechanism of the amino-thiocarbamate catalyzed enantioselective bromocyclization.

In addition to the bifunctional amino-thiocarbamate catalyst, we later discovered that dialkyl cyclic selenides are also capable chiral catalyst for the enantioselective bromoaminocyclization of olefinic amides (Scheme 11). The motivation for designing such dialkyl selenides is to overcome the low reactivity of certain olefinic amides towards catalysis by amino-thiocarbamates. While higher reactivity was observed with amino-selenothiocarbamates, however, they were found

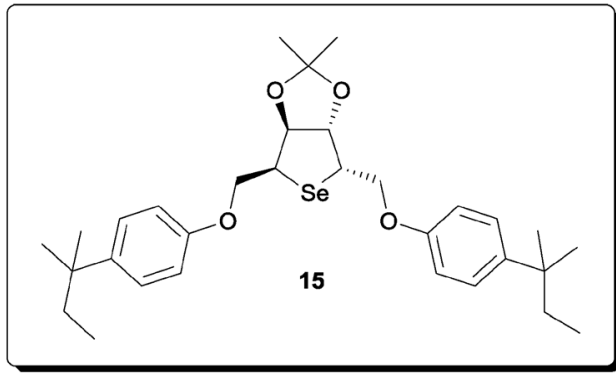

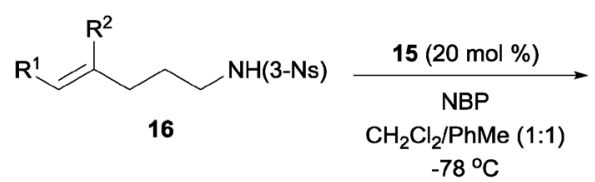

to be unstable for long term storage as well as during the reaction. $^{29}$

Building up from $d$-mannitol, cyclic selenide catalyst 15 was capable of effecting the enantioselective cyclization of tri-substituted olefinic amides. ${ }^{30}$ Interestingly, in contrast to the cinchona alkaloid based amino-thiocarbamates, the 3-nosyl amide was found to be the optimal substituent for the substrate.

The above-mentioned examples show substrates containing both an olefin and a sufficiently acidic nucleophile. Enantioselective bromoetherifications were not reported by groups using the above catalytic systems. Instead, enantioselective bromoetherifications were reported by several groups using chiral phosphoric acid derivatives.

Hennecke and co-workers reported the desymmetrization of 1,8-oct-4-ene-diols with the chiral phosphate catalyst 18 (Scheme 12). ${ }^{31}$ While modest enantioselectivity was achieved with NBS, much higher enantioselectivity was reported with $\mathrm{N}$-iodo-2-pyrrolidone.

Later, more highly enantioselective bromoetherifications were reported independently by Denmark and Shi. In Denmark's case, the use of a co-catalyst triphenylphosphine sulfide was found to be an essential requirement (Scheme 13). ${ }^{32}$ A number of substrates including 5-aryl-4-pentenols are applicable to their protocol. In their study, they observed that the $Z$-pentenols gave

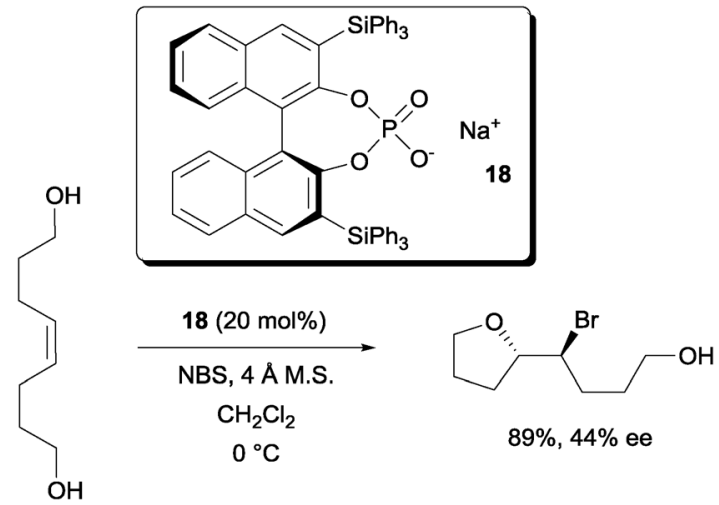

Scheme 12 Desymmetrization of 1,8-oct-4-ene diols with a chiral phosphate. 


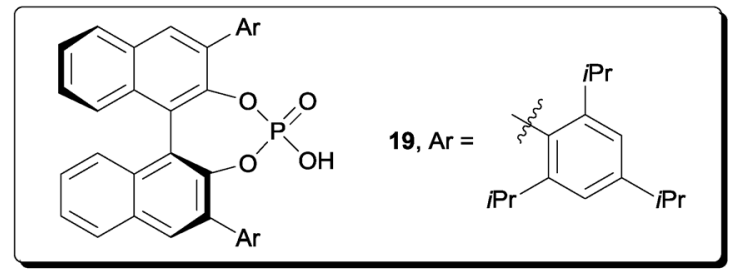<smiles>[R]C=CCCCO</smiles>

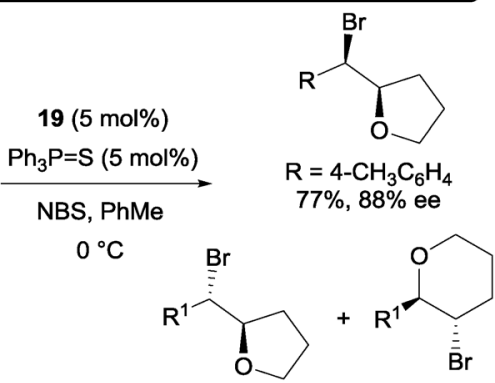

$\mathrm{R}^{1}=4-\mathrm{CH}_{3} \mathrm{C}_{6} \mathrm{H}_{4}, 28 \%$ exo $+67 \%$ endo
$37: 63$ exolendo

$94 \%$ ee (exo); $30 \%$ ee (endo)

Scheme 13 Enantioselective bromoetherification with chiral phosphoric acid and triphenylphosphine sulphide co-catalyst.

an almost exclusive yield of the exo-cyclized bromoethers with high enantioselectivities. On the other hand, the $E$-configured pentenols gave a mixture of both exo and endo cyclized bromoethers. In terms of enantioselectivity, high ee was detected among the exo cyclized bromoethers but much reduced enantioselectivity was detected for the endo cyclized bromoethers.

Shi and co-workers utilized chiral phosphoric acid 19 as the sole catalyst (Scheme 14). ${ }^{33}$ 5-Alkyl-4-pentenols were also applicable substrates with good enantioselectivity. In addition, they were also able to apply substrates with nitrogen nucleophile to the catalytic protocol.

In another recent study, Toste et al. reported a highly enantioselective bromocyclization using chiral phosphoric acids together with a specialized DABCO derived tricationic brominating reagent. ${ }^{34}$

The final example of $N$-bromoamide induced enantioselective intramolecular bromofunctionalization in this section is the $\mathrm{N}$-bromoacetamide induced semi-pinacol rearrangement of tertiary alcohol 20 to bromoketones 21. Using a combination of cinchona alkaloid based (DHQD) ${ }_{2} \mathrm{Pyr}$ and $N$-Boc-L-phenylglycine (NBLP)

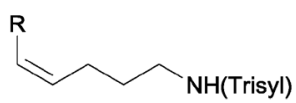<smiles>[R]C=CCCCO</smiles>

$\underset{\text { NBS, PhMe }}{\stackrel{19(5 \mathrm{~mol} \%)}{ }}$
$0^{\circ} \mathrm{C}$

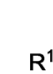

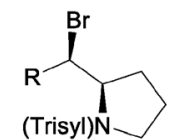

$\mathrm{R}=n-\mathrm{C}_{5} \mathrm{H}_{11}$ $77 \%, 88 \%$ ee

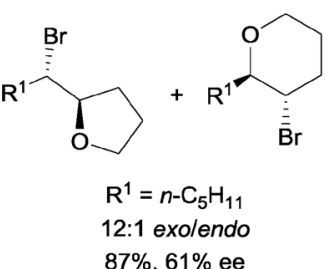

Scheme 14 Enantioselective bromoetherification with chiral phosphoric acid catalyst.

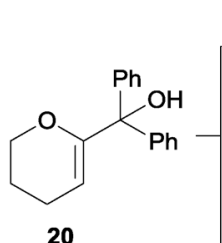

20<smiles>O=C(c1ccccc1)[C@]1(c2ccccc2)OCCC[C@@H]1Br</smiles>

(2R,3S)-21

$92 \%, 98 \%$ ee<smiles>O=C(c1ccccc1)[C@@]1([Pb])OCCC[C@H]1Br</smiles>

$(2 S, 3 R)-21$

$76 \%, 96 \%$ ee
Scheme 15 Enantioselective semi-pinacol rearrangement.

catalyst, they were able to afford $(2 R, 3 S)-21$ with good yield and enantioselectivity (Scheme 15). ${ }^{35}$ The enantioenriched $(2 S, 3 R)-21$ was obtainable by switching to (DHQ) ${ }_{2} \mathrm{Pyr}$ and $N$-Boc-D-phenylglycine (NBDP) catalyst.

\subsection{Enantioselective intermolecular bromofunctionalization of alkenes}

In comparison to enantioselective intramolecular bromofunctionalization of alkenes, the intermolecular format has proven to be a tougher problem to tackle. In a recent report, a promising enantioselective intermolecular bromoesterification was disclosed. Using chiral phosphoric acid catalyst 22, promising enantioselectivities could be obtained (Scheme 16). ${ }^{36}$ The yields were however low due to the catalyst acting as a competitive nucleophile in opening the bromonium intermediate.

Another organocatalytic approach to intermolecular bromoesterification was recently reported by Tang and co-workers. Using (DHQD) $)_{2}$ PHAL with (+)-camphor sulfonic acid (CSA) as an additive, they were able to obtain the highly enantioselective bromoesterification of a range of allylic sulfonamides (Scheme 17). ${ }^{37}$
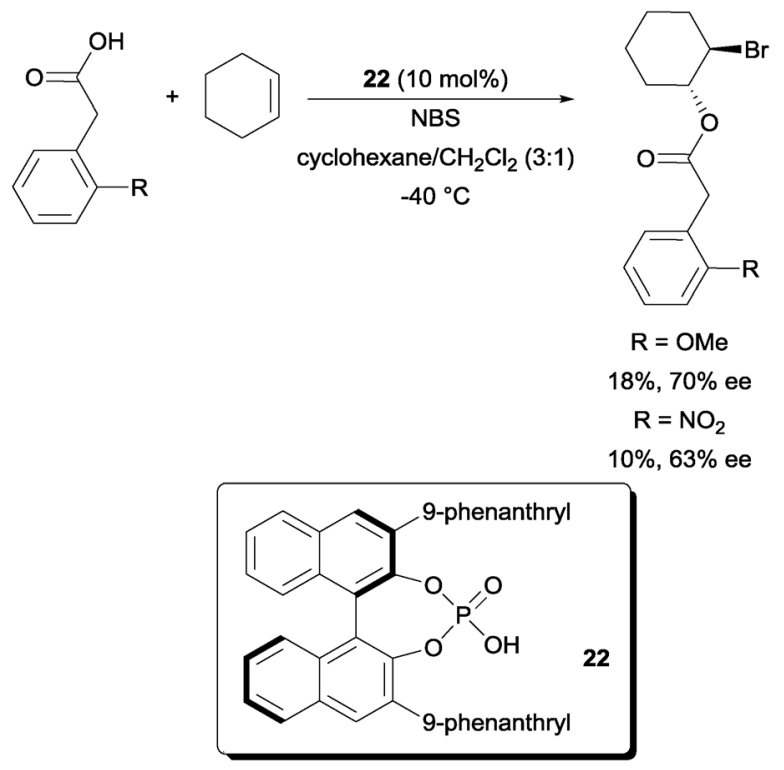

Scheme 16 Enantioselective intermolecular bromoesterification with chiral phosphoric acid. 


$$
\begin{aligned}
& \curvearrowright \mathrm{NHTf}_{\begin{array}{c}
\mathrm{NBS}(1.1 \text { equiv) } \\
(+)-\mathrm{CSA}(20 \text { mol\%) }
\end{array}}^{\frac{(\mathrm{DHQD})_{2} \mathrm{PHAL}(20 \mathrm{~mol} \%)}{\mathrm{PhCO}_{2} \mathrm{H}(1.1 \text { equiv) }}} \\
& \mathrm{CHCl}_{3}, \mathrm{rt}, 12 \mathrm{~h} \\
& \mathrm{R}=\mathrm{Ph}, 82 \%, 90 \% \text { ee } \\
& \mathrm{R}=4-\mathrm{CN}-\mathrm{C}_{6} \mathrm{H}_{4}, 79 \%, 93 \% \text { ee }
\end{aligned}
$$

Scheme 17 Enantioselective intermolecular bromoesterification with (DHQD) 2 PHAL.

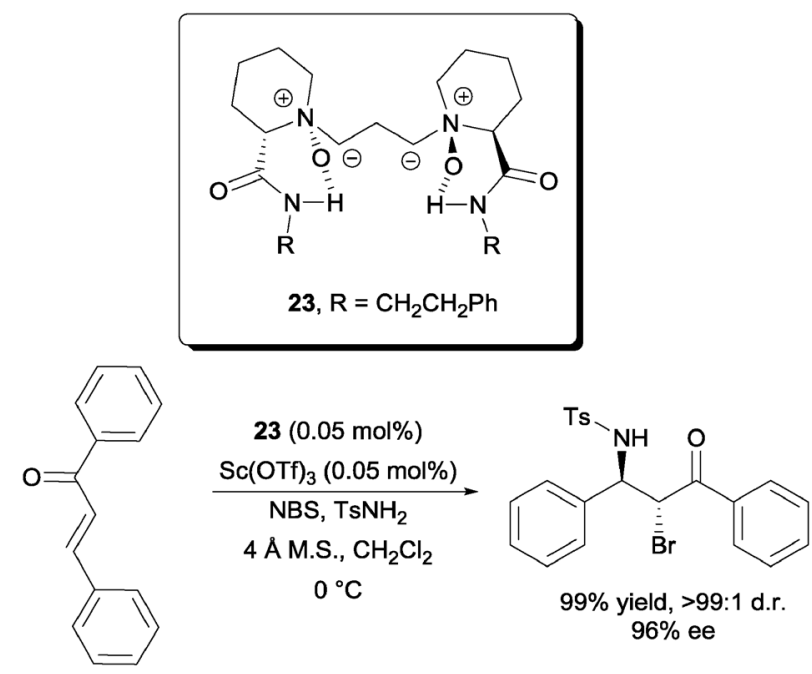

Scheme 18 Enantioselective intermolecular bromoamination of chalcone.

A highly enantioselective intermolecular bromofunctionalization was reported by Feng et al. with a scandium triflate- $N, N^{\prime}$-dioxide metal complex catalyst (Scheme 18). ${ }^{38}$ Using just $0.05 \mathrm{~mol} \%$ of the catalyst and ligand system, they were able to achieve the enantioselective bromoamination of chalcone with NBS and $\mathrm{TsNH}_{2}$. High yields and high diastereoselectivities were also achieved. It is also worth noting that successful chloro- ${ }^{39}$ and iodoamination ${ }^{40}$ of chalcones were reported by the same group.

\section{Part II - recent progress in diastereose- lective bromofunctionalization of alkenes}

Although it is mentioned in Scheme 1 that the opening of a rigid bromonium intermediate leads to an anti-relationship between the bromine and nucleophile, there has been much room for the development of novel diastereoselective bromofunctionalization of alkenes. In many cases, a Lewis acid or a Lewis base catalyst is needed to activate the brominating reagent towards such reactions. Nevertheless, recently discovered NBS-initiated cascades and multicomponent reactions (MCRs) have been found to proceed without the need for an additional catalyst. The Lewis acid catalyzed and catalyst-free MCRs will be addressed accordingly in this section.

\subsection{Diastereoselective bromofunctionalization of alkenes with a Lewis acid catalyst}

In Corey's concise synthesis of oseltamivir phosphate (Tamiflu), ${ }^{41}$ they disclosed a novel Lewis acid catalyzed haloamidation of

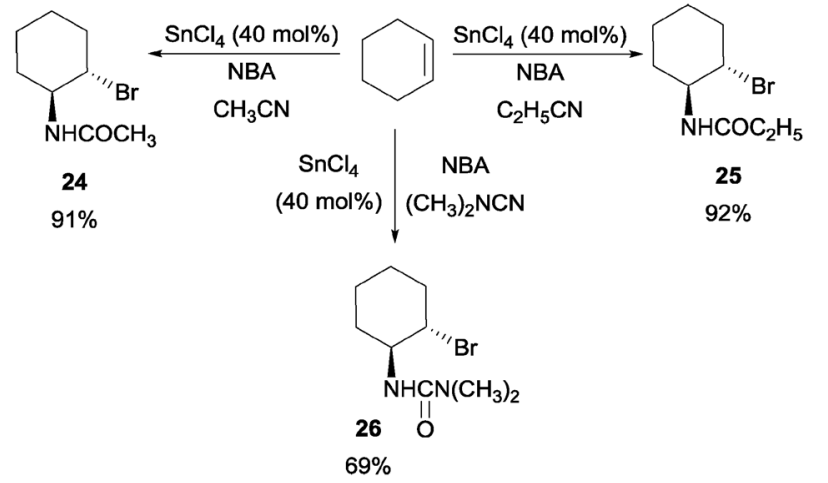

Scheme 19 Tin(IV) chloride catalyzed haloamidation of olefin.

olefins in a Ritter type fashion. It was reported that a catalytic amount of $\mathrm{SnCl}_{4}$ could catalyze the haloamidation process with NBA as the brominating source (Scheme 19). ${ }^{42}$ In addition, a variety of nitriles and olefins could be subjected to this reaction.

The mechanism was proposed to proceed via a bromonium ion ring intermediate which was opened by acetonitrile to generate intermediate $\mathbf{C}$ (Scheme 20). Attack by water on the nitrile could result in intermediate $\mathbf{D}$ which could isomerize into the bromoamide product.

Hajra et al. reported a series of metal triflate catalyzed bromofunctionalization of alkenes. In the first of their reports, they demonstrated that with $\mathrm{Zn}(\mathrm{OTf})_{2}$ as the catalyst, they could afford a series of 1,5-disubstituted tetrazoles 27 with an anti-relationship to the bromide (Scheme 21). ${ }^{43}$ Following the opening of the bromonium intermediate by the azide from

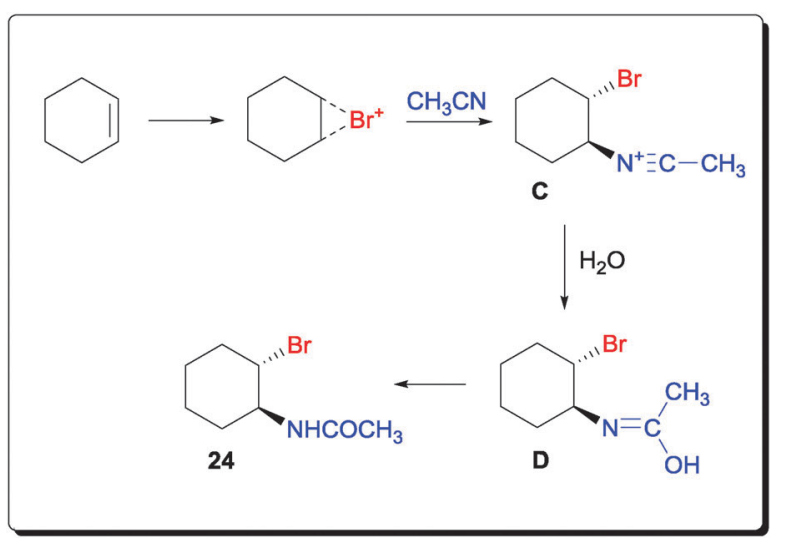

Scheme $\mathbf{2 0}$ Proposed mechanism of haloamidation of olefin.

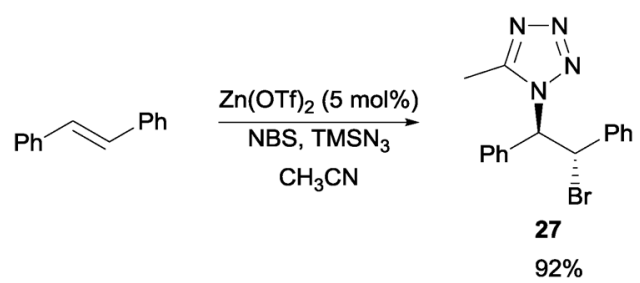

Scheme 21 NBS induced synthesis of 1,5-disubstituted tetrazoles from alkenes. 

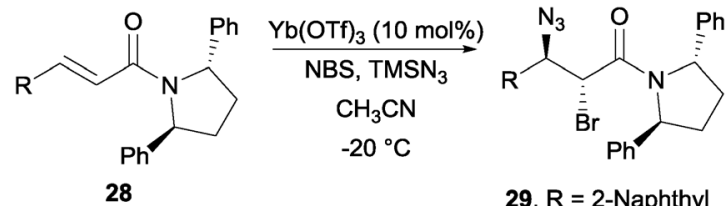

29, $\mathrm{R}=$ 2-Naphthyl

$91 \%, 94: 6 \mathrm{dr}$

Scheme 22 NBS induced bromoazidation of $\alpha, \beta$-unsaturated carboxylic acid derivatives.<smiles>COC(=O)/C=C/Br</smiles>

30

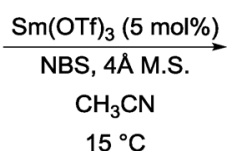<smiles>C=C(C)[C@H]([Al])C(Br)C(=O)OC</smiles>

31, $\mathrm{Ar}=4-\mathrm{MeOC}_{6} \mathrm{H}_{4}$ $91 \%$

Scheme 23 NBS promoted Friedel-Crafts alkylation of alkene.

$\mathrm{TMSN}_{3}$, assemblage into tetrazole ensued with the acetonitrile solvent. The reaction was found to be applicable to a range of olefins as well as a number of nitrile sources.

With $\mathrm{Yb}(\mathrm{OTf})_{3}$ as the catalyst, Hajra and co-workers were able to achieve a highly diastereoselective bromoazidation of $\alpha, \beta$-unsaturated carboxylic acid derivatives with the aid of chiral auxiliaries such as $(2 R)$-bornane sultam and $(2 S, 5 S)$-diphenyl pyrrolidine (Scheme 22). ${ }^{44}$

Another interesting example was achieved via the use of $\mathrm{Sm}(\mathrm{OTf})_{3}$ as the catalyst for a NBS promoted Friedel-Crafts alkylation of alkenes (Scheme 23). ${ }^{45}$ With electron-rich aromatic rings including toluene, $o$-xylene, anisole, 1,2-dimethoxy benzene, and 1,2,3-trimethoxy benzene, they were able to achieve this transformation with a number of olefinic partners.

Lastly, with $\mathrm{Cu}(\mathrm{OTf})_{2}$ or $\mathrm{Zn}(\mathrm{OTf})_{2}$ Hajra and co-workers were able to achieve the stereoselective one-pot synthesis of oxazolines via a bromoamide intermediate (Scheme 24 ). ${ }^{46}$ In a process similar

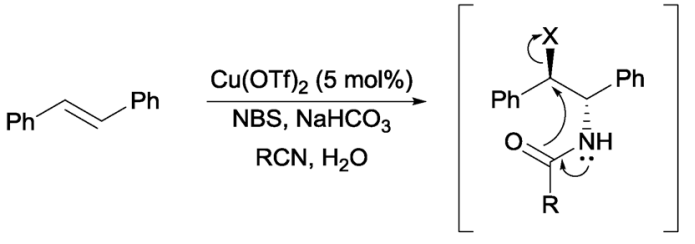

$$
\begin{aligned}
& \overbrace{\mathrm{Ph}}^{\mathrm{Ph}} \\
& \text { 32a, } R=M e, 62 \% \\
& \text { 32b, } R=P h, 65 \%
\end{aligned}
$$

Scheme 24 NBS promoted stereoselective one-pot synthesis of oxazolines. to Corey's haloamidation, the bromoamides were able to convert into oxazolines in the presence of $\mathrm{NaHCO}_{3}$ and water.

\subsection{NBS initiated cyclic ether opening cascades}

In Braddock and co-workers' study of model obtusallene systems, they discovered that NBS can be utilized to initiate a transannular oxonium ion formation-fragmentation reaction (Scheme 25). ${ }^{47}$ When the macrocyclic compound 33 was treated with NBS, the reaction proceeded in the presence of a nucleophile, for example acetic acid. Addition of $1 \mathrm{~mol} \%$ of tetramethylguanidine (TMG) was found to be necessary. In addition, it was also found that bromine and water can also serve as the nucleophilic partners for this reaction.

In a later study, Jamison et al. found that NBS can initiate an epoxide-opening cascade to afford products leading to the total synthesis of ent-dioxepandehydrothyrsiferol (Scheme 26). ${ }^{48}$ When the poly epoxide 35 with an OBoc terminal group was treated with NBS in a highly polar solvent hexafluoroisopropanol (HFIP), the desired brominated polycycle was afforded in 36\% yield. The polycycle $\mathbf{3 6}$ was further elaborated to complete the total synthesis.

On simpler systems, Braddock and co-workers found that unlike Jamison's case ${ }^{48}$ and other earlier reports, ${ }^{49}$ the nucleophiles need not be tethered to the epoxide-containing molecules. ${ }^{50}$ Similar to their earlier study on the transannular oxonium ion formationfragmentation, they found that treatment of epoxide 37 with NBS and acetic acid with $1 \mathrm{~mol} \%$ of TMG afforded the isomeric bromoethers 38 and 39 in a 1:1 mixture with a combined yield

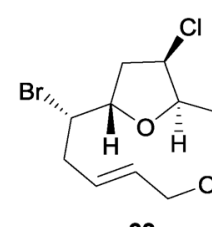

33

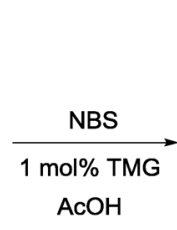

$\mathrm{AcOH}$

(1)

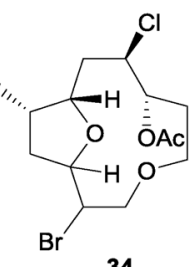

$30 \%$
Scheme 25 NBS initiated transannular oxonium ion formation-fragmentation.
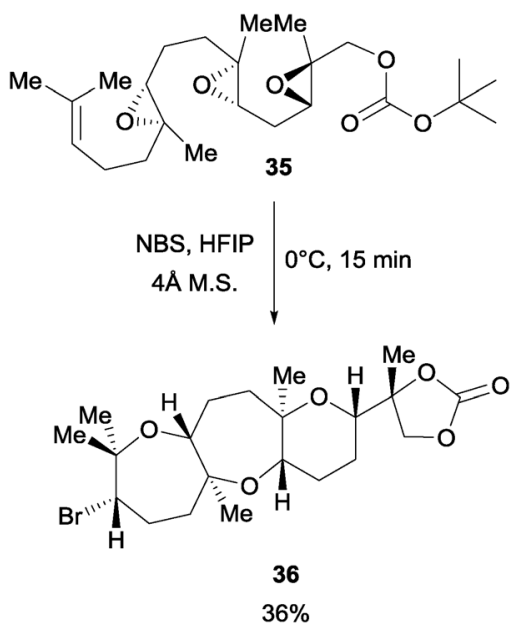

Scheme 26 NBS initiated epoxide opening cascade. 
<smiles>C1=CCCC2OC2CC1</smiles>

37

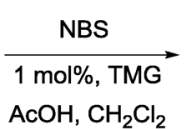

$\mathrm{AcOH}, \mathrm{CH}_{2} \mathrm{Cl}_{2}$

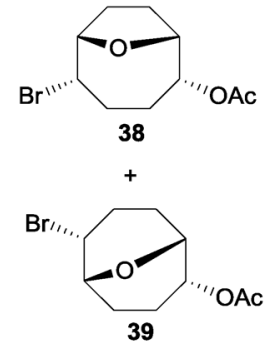

(43\% 1:1 mixture)

Scheme 27 NBS initiated epoxide opening with an external nucleophile.

of $43 \%$ (Scheme 27). While the yield of such NBS-initiated epoxide cascades was moderate, Snyder and co-workers have reported higher yielding epoxide cascades with the more reactive $\mathrm{Et}_{2} \mathrm{SBr} \cdot \mathrm{SbBrCl}_{5}$ (BDSB) reagent. ${ }^{51}$

\subsection{Catalyst free NBS initiated MCRs}

In contrast to the above-mentioned examples, our laboratory discovered a range of catalyst-free NBS-initiated multicomponent reactions (MCRs) with a variety of olefins. Such kinds of electrophilic MCRs are less reported, partly due to incompatibility of the reagents. ${ }^{52}$ In our initial conception, we envisaged the feasibility of activating olefins towards a nucleophilic attack by cyclic ethers yielding intermediate $\mathbf{E}$ (Scheme 28). The resulting oxonium intermediate $\mathbf{F}$ could then be further attacked by an amine source to yield the desired aminoalkoxylation product $\mathbf{G}^{53}$

The reaction generally works well with $\mathrm{NsNH}_{2}$ as the nucleophile and a variety of olefins and cyclic ethers were found to be tolerated. The products obtained had good positional(Markonikov-type) as well as stereo-selectivities. In a demonstration of the usefulness of the reaction, the one-pot assembly of trans-1-phenyl-1-propene, $\mathrm{NsNH}_{2}$, ethylene oxide, and NBS resulted in the formation of the key intermediate $\mathbf{4 0}$ (Scheme 29). With further synthetic manipulation, $( \pm)$-phenmetrazine and Bontril $^{\mathrm{TM}}$ were both obtained with good yields. ${ }^{54}$

In addition to using cyclic ethers, nitriles and cyanimides could be used in place to afford a range of imidazoline ${ }^{55}$ and guanidine, ${ }^{56}$ respectively. The MCRs proceed via the one-pot assembly of olefin, $\mathrm{NBS}, \mathrm{NsNH}_{2}$, and nitriles or cyanimides to
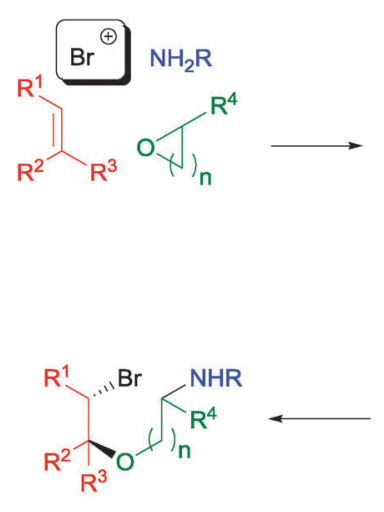

G

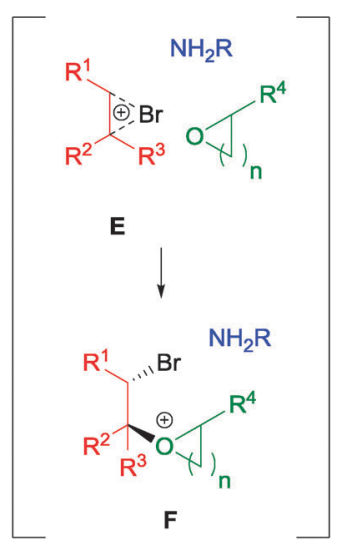

Scheme 28 Proposed mechanism of NBS initiated aminoalkoxylation of olefins.

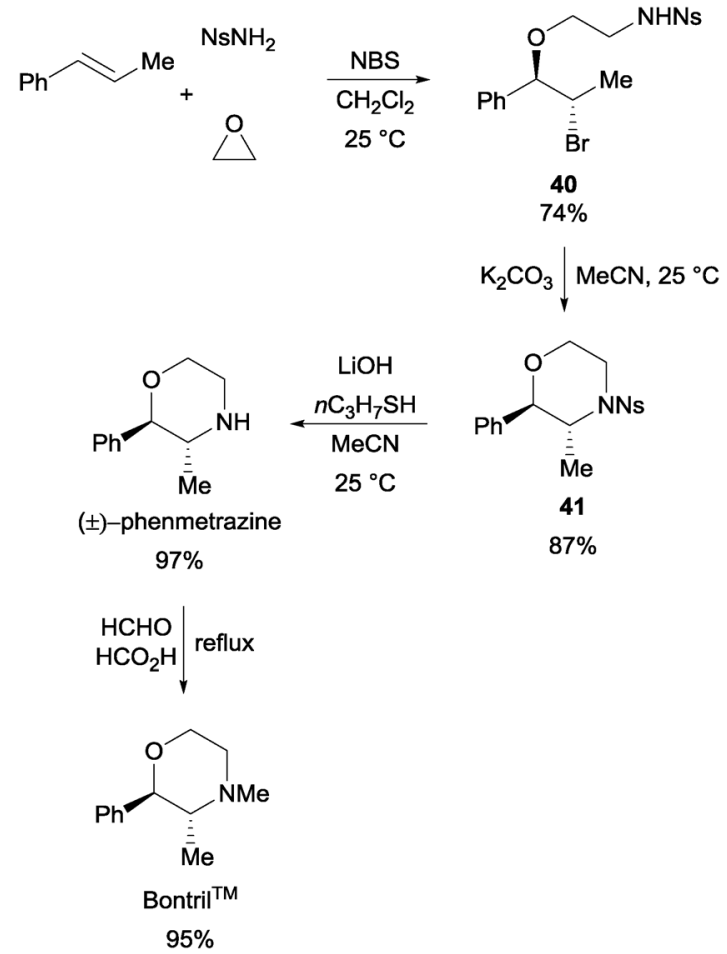

Scheme 29 Synthesis of pharmaceutically relevant molecules via the NBS initiated MCR.

afford the respective bromoamides (Scheme 30). Subsequent intramolecular displacement of the bromides in the bromoamides and cyclization (either in situ or by further refluxing the bromoamides) led to the formation of imidazolines and guanidines.

Oxygen nucleophiles in the form of carboxylic acids were also found to be feasible reaction partners in this type of MCR. In a demonstration, the one-pot reaction of cyclohexene, NBS, acetic acid, and THF was found to yield the alkoxylether product 44 in good yield (Scheme 31). ${ }^{57}$ Likewise for this reaction, a variety of olefins, cyclic ethers as well as carboxylic acids were found to be applicable to this MCR protocol.
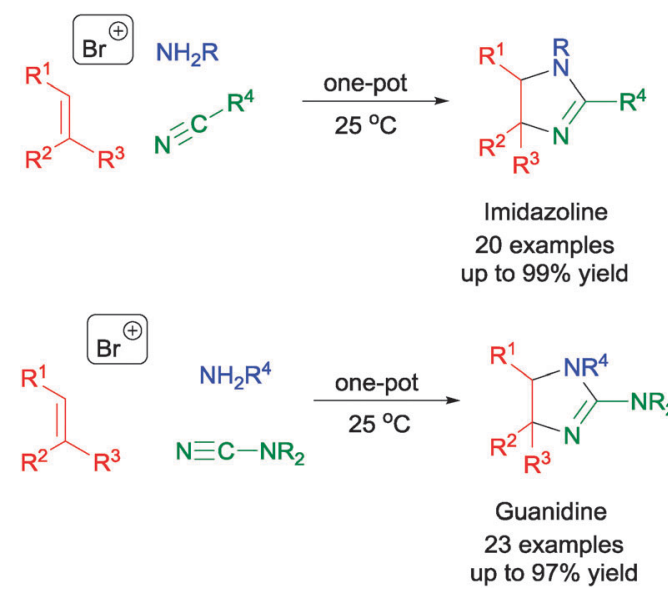

Scheme 30 NBS initiated MCR in the synthesis of imidazolines and guanidines. 
<smiles>O=C1CCC(=O)N1Br</smiles><smiles>O=C(O)C(O)c1ccccc1</smiles><smiles>[R]C(Br)C([R])([R])OCC(C)(C)COC(=O)C(O)c1ccccc1</smiles><smiles>O=C(O)[C+]1CCCCC1</smiles>

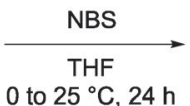

0 to $25^{\circ} \mathrm{C}, 24 \mathrm{~h}$

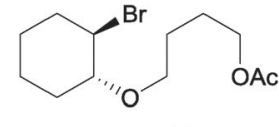

44
Scheme 31 NBS initiated alkoxyetherification of olefin.<smiles>[R]C([R])=C</smiles>

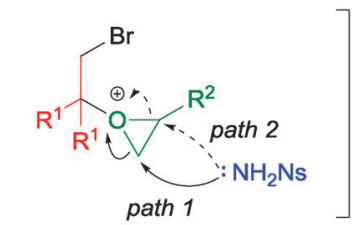
$\stackrel{\text { path } 1}{\longrightarrow}$<smiles>[R]C(CN)OC([R])([R])CBr</smiles><smiles>[R]C1C[N-]CC([R7])([R])O1</smiles>

$\stackrel{\text { path } 2}{\longrightarrow}$<smiles>[R]C(COC([R])([R])CBr)NS([R])(C)C</smiles>

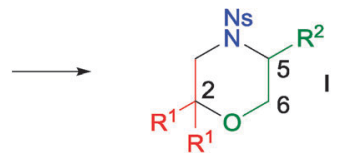

Scheme 32 Proposed mechanism of MCR with mono-substituted epoxide.

A more challenging MCR involving mono-substituted epoxides was also tackled by our group (Scheme 32 ). ${ }^{58}$ In this reaction, after the formation of the bromo oxonium intermediate, the subsequent oxonium ring opening can proceed via the anti-Markovnikov (path 1) or the Markovnikov (path 2) pathways. In our investigation, we discovered that propylene oxide gave no regioselectivity $(\mathbf{H}: \mathbf{I}=1: 1)$ despite the high reaction yield. Other bulky mono-substituted epoxides (e.g. $\mathrm{R}^{2}=$ OTBDPS) resulted in low regioselectivity as well. Nonetheless, it was found that when electron-deficient epoxides like epichlorohydrin and epibromohydrin were used, good regioselectivities were achieved.

In a demonstration of the synthetic utility, enantiopure $(R)$-epichlorohydrin could assemble with olefin $45, \mathrm{NBS}$, and $\mathrm{NsNH}_{2}$ (Scheme 33). Upon treatment with base, enantiopure 2,2,6-trisubstituted morpholine $\mathbf{4 6}$ was obtained with good yield.

More recently, it was also discovered that sufficiently acidic phenols could be used as a nucleophile in this MCR to generate a phenoxyetherification product like 47 (Scheme 34).${ }^{59}$ Crucially, it was discovered that when relatively electron-rich phenols, including 4-methyl, 4-tert-butyl, and 2,6-dibromo-4-methyl

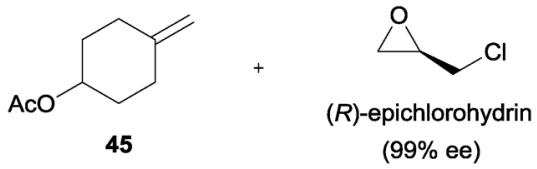

$\mathrm{NBS}, \mathrm{NsNH}_{2}$ then $\mathrm{K}_{2} \mathrm{CO}_{3}, \mathrm{MeCN}$

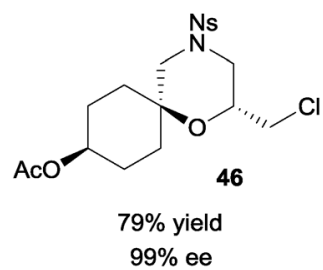

Scheme 33 Enantioselective synthesis of 2,2,6-trisubstituted morpholine via NBS initiated MCR.
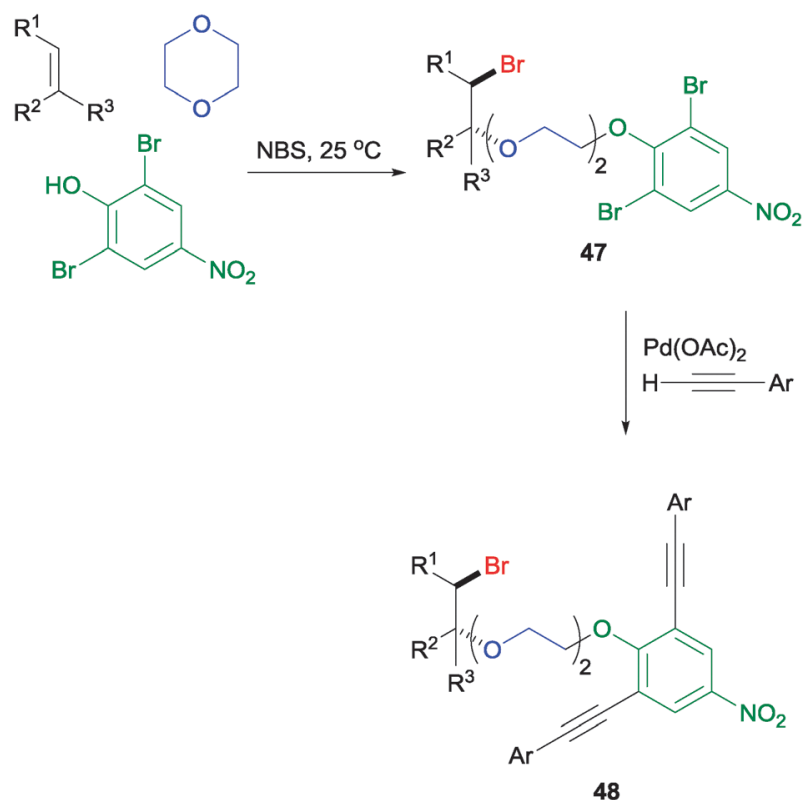

Scheme 34 NBS initiated phenoxyetherification MCR and its application.

phenols, were subjected to the reaction, no desired product was detected. This result is consistent with our previous observation in which the acidic proton plays a crucial role in the MCR, potentially acting as the NBS activator. Again, the MCR tolerates a range of olefinic partners as well as ether partners for a wider reaction scope. More interestingly, the dibromides on the aromatic ring can undergo a double Sonogashira coupling to generate compound 48, which may serve as ligands to self-assembly spherical complexes that are potentially applicable to the construction of high-density datastorage materials. ${ }^{60}$

\section{Conclusion}

This feature article has charted the recent advances of the use of $N$-bromoamide in the bromofunctionalization of alkenes. In the course of the article, the recent flurry of reports in the area 
of enantioselective bromofunctionalization of alkenes was highlighted. In these reports the previously noted difficulties of enantioselective bromofunctionalization of alkenes have been somewhat surmounted. However there is still room for expansion of reaction scope especially for the intermolecular enantioselective bromofunctionalization of alkenes. The arena of diastereoselective bromofunctionalization of alkenes still has much room for creative exploration. Examples of the use of brominating reagents for cyclic ether opening cascades and electrophilic multicomponent reactions are just some of the examples highlighted in this article. We believe other bromine initiated cascades may be explored in the future. Additionally, a clear mechanistic understanding of the described bromofunctionalization reactions has yet to emerge. Further investigation into the origin of the enantioselectivity for the asymmetric bromofunctionalization of alkenes will be especially useful for future development.

We thank the financial support from National University of Singapore (Grant No. 143-000-509-112), ASTAR-Public Sector Funding (Grant no. 143-000-536-305), and GSK-EDB. C. K. Tan would like to acknowledge President's Graduate Fellowship for the sponsoring of his PhD scholarship.

\section{Notes and references}

1 J. S. Pizey, Synthetic Reagents, Wiley, New York, 1974, vol. 2, p. 1.

2 F. A. Carey and R. J. Sundberg, Advanced Organic Chemistry Part A: Structure and Mechanisms, Springer, New York, 5th edn, 2007, pp. $476-482$.

3 D. S. Noyce and J. A. Virgilio, J. Org. Chem., 1972, 37, 2643-2647.

4 (a) C. Djerassi, Chem. Rev., 1948, 43, 271-317; (b) L. Horner and E. H. Winkelmann, Angew. Chem., 1959, 71, 349-365.

5 (a) T. F. Corbin, R. C. Hahn and H. Shecter, Org. Synth., 1973, 5, 328; (b) A. Kalir, Org. Synth., 1973, 5, 825.

6 (a) M. Yamaura, T. Suzuki, H. Hashimoto, J. Yoshimura and C. Shin, Bull. Chem. Soc. Jpn., 1985, 58, 2812-2920; (b) Z. Lidert and S. Gronowitz, Synthesis, 1980, 322-324.

7 R. H. Mitchell, Y.-H. Lai and R. V. Williams, J. Org. Chem., 1979, 44, 4733-4735.

8 (a) H. M. Gilow and D. E. Burton, J. Org. Chem., 1981, 46, 2221-2225; (b) S. Martina, V. Enkelmann, G. Wegner and A.-D. Schlüter, Synthesis, 1991, 613-615; (c) R. M. Kellogg, A. P. Schaap, E. T. Harper and H. Wynberg, J. Org. Chem., 1968, 33, 2902-2909; (d) Y. Goldberg and H. Alper, J. Org. Chem., 1993, 58, 3072-3075.

9 (a) G. A. Olah and J. M. Bollinger, J. Am. Chem. Soc., 1967, 89, 4744-4752; (b) G. A. Olah and J. M. Bollinger, J. Am. Chem. Soc., 1968, 90, 947-953; (c) G. A. Olah, J. M. Bollinger and J. Brinich, J. Am. Chem. Soc., 1968, 90, 2587-2594.

10 F. A. Carey and R. J. Sundberg, Advanced Organic Chemistry Part A: Structure and Mechanisms, Springer, New York, 5th edn, 2007, pp. $473-497$.

11 (a) A. Castellanos and S. P. Fletcher, Chem.-Eur. J., 2011, 17, 5766-5776; (b) S. A. Snyder, D. S. Treitler and A. P. Brucks, Aldrichimica Acta, 2011, 44, 27-40; (c) C. K. Tan, Z. Ling and Y.-Y. Yeung, Synlett, 2011, 1335-1339; (d) U. Hennecke, Chem.-Asian J., 2012, 7, 456-465; (e) S. E. Denmark, W. E. Kuester and M. T. Burk, Angew. Chem., Int. $E d ., 2012$, 51, 10938-10953; $(f)$ K. Murai and H. Fujioka, Heterocycles, 2013, 87, 763-805.

12 (a) R. S. Brown, R. W. Nagorski, A. J. Bennet, R. E. D. McClung, G. H. M. Aarts, M. Klobukowski, R. McDonald and B. D. Santarsiero, J. Am. Chem. Soc., 1994, 116, 2448-2456; (b) A. A. Neverov and R. S. Brown, J. Org. Chem., 1996, 61, 962-968; (c) R. S. Brown, Acc. Chem. Res., 1997, 30, 131-137.

13 S. E. Denmark, M. T. Burk and A. J. Hoover, J. Am. Chem. Soc., 2010, 132, 1232-1233.

14 (a) D. C. Whitehead, R. Yousefi, A. Jaganathan and B. Borhan, J. Am. Chem. Soc., 2010, 132, 3298-3300; (b) R. Yousefi, D. C. Whitehead,
J. M. Mueller, R. J. Staples and B. Borhan, Org. Lett., 2011, 113, 608-611; (c) A. Jaganathan, A. Garzan, D. C. Whitehead, R. J. Staples and B. Borhan, Angew. Chem., Int. Ed., 2011, 50, 2593-2596; (d) K. C. Nicolaou, N. L. Simmons, Y. Ying, P. M. Heretsch and J. S. Chen, J. Am. Chem. Soc., 2011, 133, 8134-8137.

15 (a) G. E. Veitch and E. N. Jacobsen, Angew. Chem., Int. Ed., 2010, 49, 7332-7335; (b) M. C. Dobish and J. N. Johnston, J. Am. Chem. Soc., 2012, 134, 6068-6071; (c) C. S. Brindle, C. S. Yeung and E. N. Jacobsen, Chem. Sci., 2013, 4, 2100-2104.

16 (a) W. Zhang, S. Zheng, N. Liu, J. B. Werness, I. A. Guzei and W. Tang, J. Am. Chem. Soc., 2010, 132, 3664-3665; (b) W. Zhang, N. Liu, C. M. Schienebec, K. Decloux, S. Zheng, J. B. Werness and W. Tang, Chem.-Eur. J., 2012, 18, 7296-7305.

17 W. Zhang, H. Xu, H. Xu and W. Tang, J. Am. Chem. Soc., 2009, 131, 3832-3833.

18 (a) K. Murai, T. Matsushita, A. Nakamura, S. Fukushima, M. Shimura and H. Fujioka, Angew. Chem., Int. Ed., 2010, 49, 9174-9177; (b) K. Murai, A. Nakamura, T. Matsushita, M. Shimura and H. Fujioka, Chem.-Eur. J., 2012, 18, 8448-8453.

19 L. Zhou, C. K. Tan, X. Jiang, F. Chen and Y.-Y. Yeung, J. Am. Chem. Soc., 2010, 132, 15474-15476.

20 C. K. Tan, L. Zhou and Y.-Y. Yeung, Org. Lett., 2011, 13, 2738-2741. 21 C. K. Tan, C. Le and Y.-Y. Yeung, Chem. Commun., 2012, 48, 5793-5795. 22 J. Chen, L. Zhou, C. K. Tan and Y.-Y. Yeung, J. Org. Chem., 2012, 77, 999-1009.

23 X. Jiang, C. K. Tan, L. Zhou and Y.-Y. Yeung, Angew. Chem., Int. Ed., 2012, 51, 7771-7775.

24 (a) D. A. Mulholland, K. McFarland and R. Randrianarivelojoisa, Biochem. Syst. Ecol., 2006, 34, 365-369; (b) I. Čorić, S. Müller and B. List, J. Am. Chem. Soc., 2010, 132, 17370-17373.

25 (a) L. Zhou, J. Chen, C. K. Tan and Y.-Y. Yeung, J. Am. Chem. Soc., 2011, 133, 9164-9167; (b) J. Chen, L. Zhou and Y.-Y. Yeung, Org. Biomol. Chem., 2012, 10, 3808-3811; (c) L. Zhou, D. W. Tay, J. Chen, G. Y. C. Leung and Y.-Y. Yeung, Chem. Commun., 2013, 49, 4412-4414, DOI: 10.1039/c2cc36578b.

26 (a) V. Gutmann, Coord. Chem. Rev., 1975, 15, 207-237; (b) V. Gutmann, The Donor-Acceptor Approach to Molecular Interactions, Plenum, New York, 1978; (c) S. E. Denmark and G. L. Beutner, Angew. Chem., Int. Ed., 2008, 47, 1560-1638.

27 (a) P. D. Boyle and S. M. Godfrey, Coord. Chem. Rev., 2001, 233, 265-299; (b) J. L. Dutton, G. J. Farrar, M. J. Sgro, T. L. Battista and P. J. Ragogna, Chem.-Eur. J., 2009, 15, 10263-10271.

28 S. E. Denmark and M. T. Burk, Proc. Natl. Acad. Sci. U. S. A., 2010, 107, 20655-20660.

29 C. K. Tan, F. Chen and Y.-Y. Yeung, Tetrahedron Lett., 2011, 52, 4892-4895.

30 F. Chen, C. K. Tan and Y.-Y. Yeung, J. Am. Chem. Soc., 2013, 135, 1232-1235.

31 U. Hennecke, C. H. Müller and R. Fröhlich, Org. Lett., 2011, 13, 860-863.

32 S. E. Denmark and M. T. Burk, Org. Lett., 2012, 14, 256-259.

33 D. Huang, H. Wang, F. Xue, H. Guan, L. Li, X. Peng and Y. Shi, Org. Lett., 2011, 13, 6350-6353.

34 Y.-M. Wang, J. Wu, C. Hoong, V. Rauniyar and F. D. Toste, J. Am. Chem. Soc., 2012, 134, 12928-12931.

35 Z.-M. Chen, Q.-W. Zhang, Z.-H. Chen, H. Li, Y.-Q. Tu, F.-M. Zhang and J.-M. Tian, J. Am. Chem. Soc., 2011, 133, 8818-8821.

36 G.-X. Li, Q.-Q. Fu, X.-M. Zhang, J. Jiang and Z. Tang, Tetrahedron: Asymmetry, 2012, 23, 245-251.

37 W. Zhang, N. Liu, C. M. Schienebeck, X. Zhou, I. I. Izhar, I. A. Guzei and W. Tang, Chem. Sci., 2013, 4, 2652-2656, DOI: 10.1039/ c3sc50446h.

38 Y. Cai, X. Liu, Y. Hui, J. Jiang, W. Wang, W. Chen, L. Lin and X. Feng, Angew. Chem., Int. Ed., 2010, 49, 6160-6164.

39 Y. Cai, X. Liu, J. Jiang, W. Chen, L. Lin and X. Feng, J. Am. Chem. Soc., 2011, 133, 5636-5639.

40 Y. Cai, X. Liu, J. Li, W. Chen, W. Wang, L. Lin and X. Feng, Chem.-Eur. J., 2011, 17, 14916-14921.

41 Y.-Y. Yeung, S. Hong and E. J. Corey, J. Am. Chem. Soc., 2006, 128, 6310-6311.

42 Y.-Y. Yeung, X. Gao and E. J. Corey, J. Am. Chem. Soc., 2006, 128, 9644-9645.

43 S. Hajra, D. Sinha and M. Bhowmick, J. Org. Chem., 2007, 72, 1852-1855.

44 S. Hajra, M. Bhowmick and D. Sinha, J. Org. Chem., 2006, 71, 9237-9240. 
45 S. Hajra, B. Majit and S. Bar, Org. Lett., 2007, 9, 2783-2786. 46 S. Hajra, S. Bar, D. Sinha and B. Majit, J. Org. Chem., 2008, 73, 4320-4322. 47 C. D. Braddock, D. S. Millan, Y. Pérez-Fuertes, R. H. Pouwer, R. N. Sheppard, S. Solanki and A. J. P. White, J. Org. Chem., 2009, 74, 1835-1841.

48 J. Tanuwidjaja, S.-S. Ng and T. F. Jamison, J. Am. Chem. Soc., 2009, 131, 12084-12085.

49 (a) S. G. Davies, M. E. C. Polywka and S. E. Thomas, Tetrahedron Lett., 1985, 26, 1461-1464; (b) S. G. Davies, M. E. C. Polywka and S. E. Thomas, J. Chem. Soc., Perkin Trans. 1, 1986, 1277-1282; (c) F. Bravo, F. E. McDonald, W. A. Neiwert and K. I. Hardcastle, Org. Lett., 2004, 6, 4487-4489.

50 K. J. Bonney, C. D. Braddock, A. J. P. White and M. Yaqoob, J. Org. Chem., 2011, 76, 97-104.

51 (a) S. A. Snyder, D. S. Treitler, A. P. Brucks and W. Sattler, J. Am. Chem. Soc., 2011, 133, 15898-15901; (b) S. A. Snyder, A. P. Brucks, D. S. Treitler and I. Moga, J. Am. Chem. Soc., 2012, 134, 17714-17721.

52 (a) Y. A. Serguchev, M. V. Ponomarenko, L. F. Lourie and A. N. Chernega, J. Fluorine Chem., 2003, 123, 207-215; (b) V. Nair, R. S. Menon, P. B. Beneesh, V. Sreekumar and S. Bindu, Org. Lett.,
2004, 6, 767-769; (c) V. Nair, P. B. Beneesh, V. Sreekumar, S. Bindu, R. S. Menon and A. Deepthi, Tetrahedron Lett., 2005, 46, 201-203; (d) T. L. Church, C. M. Byrne, E. B. Lobkovsky and G. W. Coates, J. Am. Chem. Soc., 2007, 129, 8156-8162; (e) T. Abe, H. Takeda, Y. Miwa, K. Yamada, R. Yanada and M. Ishikura, Helv. Chim. Acta, 2010, 93, 233-241.

53 L. Zhou, C. K. Tan, J. Zhou and Y.-Y. Yeung, J. Am. Chem. Soc., 2010, 132, 10245-10247.

54 R. B. Rothman and M. H. Baumann, Curr. Top. Med. Chem., 2006, 6, 1846.

55 L. Zhou, J. Zhou, C. K. Tan, J. Chen and Y.-Y. Yeung, Org. Lett., 2011, 13, 2448-2451.

56 L. Zhou, J. Chen, J. Zhou and Y.-Y. Yeung, Org. Lett., 2011, 13, 5804-5807.

57 J. Chen, S. Chng, L. Zhou and Y.-Y. Yeung, Org. Lett., 2011, 13, 6456-6459.

58 J. Zhou, L. Zhou and Y.-Y. Yeung, Org. Lett., 2012, 14, 5250-5253.

59 Z. Ke and Y.-Y. Yeung, Org. Lett., 2013, 15, 1906-1909.

60 T. Murase, S. Sato and M. Fujita, Angew. Chem., Int. Ed., 2007, 46, 1083-1085. 